\title{
PREMIO SALARIAL EN EL SECTOR PÚBLICO: EVOLUCIÓN EN EL PERIODO 1990-2017
}

\author{
Adolfo Fuentes \\ Centro de Estudios Públicos \\ Rodrigo Vergara \\ Centro de Estudios Públicos
}

\begin{abstract}
RESUMEN: Este artículo estima el premio salarial del sector público para el período 1990-2017, utilizando los datos de la encuesta Casen. Se entiende el premio salarial como la diferencia entre el salario del sector privado y público que no es explicado por observables de los trabajadores en ambos sectores. Los resultados muestran que dicho premio ha aumentado de 1,5 por ciento en 1990 a 20 por ciento en 2017. Esto es, en la actualidad un empleado del sector público gana un 20 por ciento más que uno del sector privado con similares características observables. La descomposición de Blinder-Oaxaca arroja que el premio pasó de representar un 5 por ciento de la diferencia salarial entre ambos sectores en 1990 (el otro 95 por ciento se explicaba por observables) a un 48 por ciento en 2017. Las estimaciones a nivel de percentiles indican que en la década de 1990 los mayores premios eran para los ingresos más bajos, mientras que en la actualidad los premios son similares para los distintos niveles de ingreso. Se observa también que la política salarial del sector público tiende a dar un mayor incentivo a las mujeres
\end{abstract}

Adolfo Fuentes. Economista de la Universidad de Chile. Investigador asistente en el Centro de Estudios Públicos (CEP). Email: afuentes@cepchile.cl.

Rodrigo Vergara. Investigador senior en el CEP. Investigador asociado del Centro Mossavar-Rahmani de la Escuela de Gobierno (Kennedy) de Harvard University. Email: rvergara@cepchile.cl.

Los autores agradecen los valiosos comentarios de Hernán Herrera, Claudia Martínez y de los árbitros anónimos de Estudios Públicos. 
y a personas con estudios técnicos y universitarios, sin importar su edad. Para verificar la robustez de los resultados se hacen comparaciones con estimaciones basadas en la Encuesta Suplementaria de Ingresos del INE para el período 2010-2017.

Palabras clave: empleo público, política de remuneraciones, premio salarial sector público, evolución brecha salarial, Casen, ESI.

RECIBIDO: septiembre 2018; ACEPTADO: noviembre 2018.

\title{
THE PUBLIC SECTOR WAGE PREMIUM AND ITS EVOLUTION IN THE PERIOD 1990-2017
}

\begin{abstract}
This article uses data from Chile's National Socioeconomic Survey (CASEN) to estimate the public sector wage premium for the period 1990-2017. By the wage premium is meant the difference between private and public sector wages that is not explained by worker observables in the two sectors. The findings show this premium to have increased from 1.5 per cent in 1990 to 20 per cent in 2017. In other words, a public sector employee now earns 20 per cent more than a private sector one with similar observable characteristics. The Blinder-Oaxaca decomposition indicates that the premium rose from 5 per cent of the wage difference between the two sectors in 1990 (the other 95 per cent was explained by observables) to 48 per cent in 2017. The percentile-level estimates show that in the 1990s the largest premiums were for the lowest incomes, while today's premiums are similar across the different income levels. It is also observed that public sector wage policy tends to particularly reward women and people with a technical or university education, irrespective of age. To check the robustness of the findings, they are compared with estimates based on the National Institute of Statistics (INE) Supplemental Income Survey for the period 2010-2017.
\end{abstract}

KEYwords: public sector employment, remuneration policy, public sector wage premium, evolution of the wage gap, CASEN, Supplemental Income Survey.

ReCEIVED: September 2018; ACCEPTED: November 2018.

\section{INTRODUCCIÓN}

$E$ n Chile, todos los años, se negocia entre el gobierno y las cúpulas sindicales el reajuste salarial de los empleados que trabajan en el sector público. Los criterios para este reajuste tienden a basarse en la inflación, el crecimiento y la capacidad fiscal del momento, con negociaciones que se dan en un contexto tanto político como técnico. 
En la práctica, estas negociaciones han concluido con reajustes que han significado que los salarios públicos crezcan a un ritmo mayor que los salarios privados, lo que ha generado un premio para los primeros.

Varios autores han medido dicho premio en Chile, para diversos periodos y con diversas metodologías. Cerda (2016), Navarro y Selman (2014) y Gallegos et al. (2011) son algunos de los más recientes. La medición de este premio es muy relevante para la política pública, ya que hay una variada gama de temas asociados a éste como gasto fiscal, ${ }^{1}$ productividad en el sector y percepción de captura del Estado, entre otros. Sin embargo, se trata de estudios sobre años en particular o sobre períodos cortos, de forma que no permiten un análisis exhaustivo de la política de remuneraciones del sector público en el tiempo.

Este artículo tiene el objetivo principal de calcular la evolución del premio del sector público para el periodo 1990-2017. La fuente principal de datos para nuestro estudio es la encuesta Casen. Asimismo, se estimará dicho premio por nivel de ingreso. El punto es ver si los datos avalan la creencia, bastante generalizada, y verificada por Melly (2005), de que en el sector público el premio es mayor a menor nivel de ingreso. Esto implicaría, en consecuencia, que en el sector público la desigualdad de salarios sería inferior a la del sector privado.

El premio se entenderá como aquel porcentaje adicional del salario del empleado privado que, en promedio, obtiene un empleado público, tras controlar por características observables. En otras palabras, es aquel monto adicional de remuneración que recibe un empleado público en relación con un empleado del sector privado, considerando características similares de ambos empleados (es decir, controlando por factores como nivel de educación, experiencia y género, entre otros). El premio se medirá para cada uno de los años de la muestra, tanto para el promedio del sector utilizando una regresión lineal, como para percentiles de ingresos utilizando regresiones de cuantiles. Adicionalmente a esto, a modo de ver si nuestras estimaciones son robustas, descompondremos la diferencia promedio de salario entre ambos sectores con la metodología de Blinder-Oaxaca, para determinar el premio del sector, y calcular qué parte de la diferencia promedio no es explicada por observables de los individuos. Por último, veremos cómo la política de

${ }^{1}$ Balbontín et al. (2018) mencionan que, entre 2007 y 2016, el gasto en personal ha sido del orden del 20 por ciento del gasto total del gobierno central. 
remuneraciones entre ambos sectores puede generar incentivos a que determinados grupos de personas busquen trabajo en el sector público.

Una potencial crítica que se le puede hacer a un estudio de estas características es la utilización de la encuesta Casen al momento de determinar la magnitud de los premios del sector público. Esto se debe a que la importancia del sector público en dicha encuesta esta subestimada según registra Cerda (2016), a través de la comparación con los datos de la Encuesta Suplementaria de Ingreso (ESI), realizada por el Instituto Nacional de Estadísticas. Anclada a esta crítica está el hecho de que la encuesta Casen considera datos autorreportados del ingreso de los trabajadores, lo que genera un problema habitual en este tipo de encuestas a la hora de estimar ecuaciones de salarios. Se trata de un problema efectivo, que hay que tomar en cuenta. No obstante, de haber sesgo y mantenerse en el tiempo, las tendencias son una buena representación de las tendencias reales de estos premios. Por otra parte, hacemos un ejercicio similar con datos de la ESI para el periodo 20102017, que es el lapso que ambas encuestas tienen en común, y obtenemos resultados que confirman los resultados obtenidos por la Casen. Debe destacarse que, precisamente, el principal motivo para utilizar la encuesta Casen radica en la capacidad que nos provee esta encuesta de poder realizar estimaciones desde el año 1990 hasta 2017.

Para efectos de este artículo, se estudiará y se reportarán los resultados cuando se utiliza el salario o ingreso total ${ }^{2}$ y no el salario por hora. Esto se debe a varias razones. Por un lado, las horas efectivamente trabajadas son autorreportadas en la Casen, de forma que pueden estar sesgadas y alterar el salario por hora. Adicional a esto, e incluso en ausencia de sesgo, las horas efectivamente trabajadas son afectadas por las licencias médicas, ${ }^{3}$ tal como menciona Cerda (2016). Por otro lado, en Chile la regulación de salarios va mayoritariamente en función de la jornada laboral completa (45 horas a la semana actualmente), como ocurre en el caso del salario mínimo, a diferencia de Estados Unidos, donde se regula un salario mínimo por hora tra-

${ }^{2}$ En estricto rigor, se debería hablar del ingreso de los trabajadores del sector público, pero en Chile se habla del salario del sector público y se refiere al ingreso de un trabajo.

${ }^{3} \mathrm{Si}$ alguien tiene licencia médica, cuando le pregunten cuántas horas efectivamente trabajó dirá cero horas o un número menor de horas a lo trabajado habitualmente, lo que aumentará artificialmente el salario por hora. 
bajada. De todas formas, para efectos de robustez se hacen también las estimaciones para el salario por hora utilizando para su cálculo las horas efectivamente trabajadas de la encuesta Casen. A partir de ello se calcula el premio del sector público para el salario por hora. Los resultados indican una tendencia similar a la encontrada cuando se toma el ingreso total, en cinco puntos porcentuales aproximadamente. El gráfico se puede ver en el Anexo.

Nuestras estimaciones, a nivel de ingresos por ocupación principal, arrojan que el premio del sector público varía desde 1,5 por ciento en 1990 a 20 por ciento en 2017. Mientras que, a nivel de percentiles, vemos una transición de un escenario en 1990 cuando el percentil 10 obtiene un premio promedio de 19 por ciento y el percentil 90, un castigo de 26 por ciento, hacia una situación relativamente homogénea en 2017 , cuando el percentil 10 tiene un premio de 19,5 por ciento y el percentil 90, un premio de 18 por ciento. ${ }^{4}$

Los dos resultados anteriores son interesantes. Indican, por una parte, que el premio del sector público ha ido aumentando en el tiempo. Así, con el transcurrir la diferencia de salarios entre trabajadores de ambos sectores con iguales observables se ha ido ampliando. Por otra parte, indican que se ha pasado desde una situación en la que el premio de los sectores de menores ingresos era mayor que el de los sectores de mayores ingresos (en estos últimos, de hecho, había un castigo) a un premio homogéneo por nivel de ingreso. En efecto, la diferencia o premio del sector público es hoy muy similar para los distintos niveles de ingreso, cosa que no era así hasta hace unos años.

Sobre la descomposición de Blinder-Oaxaca, encontramos que su estimación del premio público (entendido como la fracción no explicada de la diferencia) es concordante con la estimación de la regresión lineal. El mayor aporte de esta descomposición es mostrar que, a medida que ha pasado el tiempo, una cada vez mayor fracción de la diferencia promedio entre ambos salarios es explicada por el simple premio, y no

${ }^{4}$ Cuando se estima utilizando datos de la ESI, aparece un premio público que va desde 20,5 por ciento en 2010 , hasta 22,7 por ciento en 2017 . Con respecto a los percentiles, encontramos en 2010 un escenario donde el percentil 10 tiene un premio promedio de 15,7 por ciento y el percentil 90 , un premio promedio de 20,2 por ciento, y en 2017 encontramos una situación homogénea para los percentiles 10 a 70 (con premios promedios de 28 y 23 por ciento, respectivamente) y para el percentil 90 , un premio promedio de 19 por ciento. 
por las características de los agentes. Así, mientras en 1990 sólo el 5 por ciento de la diferencia de salarios no era explicada por características, en 2017 esta proporción fue de 48 por ciento de la diferencia de salarios. Las estimaciones con datos de la ESI también van en esta línea, encontrando que en 2017 dicho valor fue de 53 por ciento.

Con respecto de los incentivos por determinadas características, encontramos que, a nivel de género, las mujeres poseen un incentivo mayor que los hombres a buscar empleo en el sector público, aunque éste ha ido bajando en el tiempo. En 1990 una mujer tenía el 86 por ciento de probabilidad de que existiese un trabajo mejor remunerado en el sector público, ${ }^{5}$ mientras que dicha probabilidad para un hombre era de 39 por ciento. Estas probabilidades en 2017 cambian a 91 por ciento y 82 por ciento, respectivamente. Sobre la edad, no encontramos incentivos específicos a un grupo etario, sino un aumento generalizado desde una probabilidad de 64 por ciento de que existiese un trabajo mejor remunerado en el sector público en 1990, hacia el 88 por ciento de probabilidad 6 en 2017. Destacamos, no obstante, el período entre 1998 y 2011, cuando se aprecia un claro castigo a la edad en el sector público. Por último, con respecto a los incentivos por niveles educacionales completos, observamos una situación homogénea en 1990, cuando cualquier calificación entregaba el 61 por ciento de probabilidad de que existiese un empleo mejor remunerado en el sector público. Dicha homogeneidad se rompe en 2017, cuando encontramos probabilidades de 73, 89, 95 y 98 por ciento para educación básica, educación media, educación técnica y educación universitaria, respectivamente.

Las estimaciones con datos de la ESI reafirman el incentivo a la mujer por sobre el hombre, aunque encontrando una brecha menor entre ambos. Sobre el incentivo a la edad, encontramos una convergencia similar a la observada con datos de la encuesta Casen. Finalmente, sobre los niveles educacionales completos, las estimaciones confirman que la educación técnica y universitaria tienen probabilidad casi segura de que

${ }^{5}$ Esta probabilidad, como se explicará en su correspondiente sección, se refiere a que el salario público proyectado para una persona de determinadas características sea mayor al salario privado proyectado. Es importante aclarar que no se refiere a la probabilidad de obtener un trabajo mejor remunerado, sino a la existencia de éste.

${ }^{6}$ Esta probabilidad es el promedio de los cuatro grupos. 
exista un trabajo mejor remunerado en el sector público, seguidas por la educación media y, posteriormente, la básica, aunque son distintas en la magnitud del incentivo de estas dos últimas, así como en su evolución durante los últimos ocho años.

La estructura de este artículo es la siguiente. En la segunda sección haremos una revisión bibliográfica de artículos relacionados con la medición del premio en el sector público, tanto para Chile como para otros países. En la tercera sección describiremos las bases de datos con las que contamos y presentaremos algunas estadísticas descriptivas de ellas. En la cuarta sección expondremos los modelos, las variables relacionadas y las metodologías de estimación de cada uno de ellos. En la quinta sección mostraremos los resultados relacionados con los distintos modelos a estimar. A continuación, la sexta sección presenta una discusión en torno a los resultados obtenidos y los distintos factores que pueden incidir en ellos. La séptima sección entrega las conclusiones de este artículo. Finalmente se incluyen algunos anexos.

\section{REVISIÓN DE LITERATURA}

Dentro de la literatura de premios salariales en el sector público existen variadas metodologías para poder estimar dicho valor. La forma más simple de hacerlo es como lo hace Cerda (2016), quien utiliza una estimación por mínimos cuadrados ordinarios con una dummy para el sector público. Cerda utiliza dos bases de datos, y cada una representa a un sector de la economía para el año 2015. La base pública está construida a partir de las bases de datos proporcionadas por la Ley de Transparencia en los distintos ministerios y servicios del Estado, de forma que tiene carácter censal de dicho sector. La base privada consiste en los datos de la Encuesta Suplementaria de Ingresos realizada por el Instituto Nacional de Estadísticas. Tras controlar por variables de edad, género, educación y región geográfica, obtiene que el premio público asciende a 32 por ciento para dicho año.

Otra forma de estimar este premio corresponde a la metodología de Navarro y Selman (2014). Ellos argumentan que trabajar directamente con datos de corte transversal genera que no se puedan medir no observables y que éstos sean incluidos en la estimación del premio. Para solucionar este problema, ellos realizan una estimación lineal (como 
la de Cerda) con datos de panel, utilizando los datos de la Encuesta de Protección Social (EPS) para los años 2002-2009. Su metodología consiste en realizar un procedimiento de propensity score matching (PSM), combinado con diferencias en diferencias, y luego estimar una ecuación de salarios como la de Cerda (2016) que controla por efectos fijos. Encuentran que el premio del sector público es menor al 2 por ciento, y en general no estadísticamente significativo, para el rango de la serie.

Una tercera forma de realizar esto es como lo trabajan Gallegos et al. (2011). Ellos utilizan el método de PSM, pero utilizando datos de las encuestas de hogares para varios países de Latinoamérica. ${ }^{7}$ Obtienen para Chile que la diferencia de salarios públicos y privados para el periodo 1996-2006 bordea el 40 por ciento, y que la parte no explicada por características transita desde el 3 por ciento en 1996 al 13 por ciento en 2006.

En el área internacional hay tres estudios que resultan particularmente interesantes. Melly (2005) propone una metodología de regresión por cuantiles para determinar el premio del sector público — similar a Cerda-, pero para variados tramos de la distribución de salarios. Utilizando datos de Alemania para el año 2000, determina que el sector público tiene un premio que ronda entre el 10 por ciento y el 20 por ciento para los primeros dos deciles de ingreso, y que luego es un premio negativo (un castigo) de entre 10 por ciento y 20 por ciento.

En esta misma línea, un poco más lejos va Mueller (1998), quien calcula el premio del sector público para Canadá usando tanto regresiones lineales y de cuantiles, como la descomposición de Blinder-Oaxaca. En primer lugar, encuentra resultados similares en ambos grupos de estimaciones, lo que de alguna forma valida ambos métodos de estimación. En segundo lugar, encuentra que los premios son positivos para los percentiles más bajos (cercanos a 10 por ciento para el percentil 10) y negativos para los percentiles más altos (cercanos al menos 5 por ciento para el percentil 90).

Por otro lado, Adamchik y Bedi (2000) proponen una metodología que permite entender los incentivos que genera la política de salarios públicos sobre determinados grupos de personas. Ellos realizan una estimación de máxima verosimilitud con información completa (FIML,

${ }^{7}$ Para Chile utilizan los datos de la encuesta Casen para los años 1996, 2000, 2003 y 2006. 
por sus siglas en inglés) con datos laborales de Polonia, con la intención de medir la diferencia de salarios e incentivos que ofrecía el sector público polaco. Lo interesante de esta modelación es que permite ver cómo la probabilidad de trabajar en un sector $\mathrm{u}$ otro es afectada por diversas características de los agentes y, de esta forma, ver qué agentes están siendo incentivados a buscar empleo en el sector privado. Por ejemplo, para Polonia ellos encuentran que el sector privado incentiva la búsqueda de empleo de hombres de alto nivel educacional.

\section{DATOS}

Para determinar el premio del sector público utilizaremos las encuestas Casen desde el año 1990 al año 2017, y para tener una mirada robusta a posibles sesgos utilizaremos la Encuesta Suplementaria de Ingreso (ESI) para el periodo 2010-2017. ${ }^{8}$ Ambas encuestas incorporan una variable que permite identificar el sector económico en el que trabaja el encuestado, dentro de los que se distingue el público. ${ }^{9}$ Este sector en general considera a funcionarios del poder legislativo y judicial, gobierno central, ministerios, servicios asociados y organismos de defensa del Estado. Esta clasificación excluye a funcionarios municipales, profesores, personal de salud y empleados de empresas públicas. El resto de los sectores que no pertenezcan a esta categoría serán considerados privados. Esta variable, agregada a todo el resto de la información socioeconómica de la encuesta, nos permite caracterizar correctamente el premio del sector público para cada uno de los años de la encuesta.

Según datos de la encuesta Casen, la relevancia del sector público dentro del mercado laboral ha ido en aumento. Mientras en 1990 agrupaba al 2,85 por ciento de los trabajadores, actualmente agrupa al 4,97 por ciento. El principal aumento se dio entre los años 2006 y 2009, cuando la planta pública pasó de representar el 3,53 por ciento del mercado laboral al 4,30 por ciento, lo que se observa en el cuadro 1 .

${ }^{8}$ En ambos casos, la población considerada son aquellas personas que tienen entre 15 y 59 años en el caso de las mujeres, y entre 15 y 64 años en el caso de los hombres.

${ }^{9}$ La definición exacta del sector es “Administración pública y defensa; planes de seguridad social de afiliación obligatoria". 
Cuadro 1. RELEVANCIA DEL SECTOR PÚBLICO EN EL MERCADO DEL TRABAJO SEGÚN LA CASEN

\begin{tabular}{lc}
\hline Año & Relevancia sector público (\%) \\
\hline 1990 & 2,85 \\
1992 & 3,11 \\
1994 & 3,05 \\
1996 & 3,43 \\
1998 & 3,76 \\
2000 & 3,81 \\
2003 & 3,80 \\
2006 & 3,53 \\
2009 & 4,30 \\
2011 & 4,13 \\
2013 & 4,23 \\
2015 & 5,02 \\
2017 & 4,97 \\
\hline
\end{tabular}

Fuente: Elaboración propia en base a encuestas Casen 1990-2017.

Según datos de la ESI, la relevancia del sector público dentro del mercado laboral ha presentado un leve aumento entre 2010 y 2017, siempre manteniéndose sobre el 5 por ciento. Aquí es donde observamos la subestimación de la participación del sector público por parte de la encuesta Casen, subestimación que a 2017 es de poco más de 0,6 por ciento. Esto lo vemos en el cuadro 2.

Respecto de la participación de la mujer en el sector público, vemos que ésta pasa del 27 por ciento en 1990 al 42 por ciento en 2017. Cabe destacar que el mayor impulso a la mujer se dio entre 2000 y 2009, cuando se llegó al 41 por ciento. Desde entonces la participación femenina en el sector público se ha mantenido relativamente estable, lo que se puede observar en el cuadro $3 .^{10}$

Lo anterior concuerda con datos de la ESI, en donde podemos apreciar el mismo nivel de estancamiento que se observa desde 2009

${ }^{10}$ Se debe tener en consideración que la porción mayoritaria de los hombres se produce por la inclusión de los órganos de defensa. Balbontín et al. (2018) muestran que a nivel de trabajadores civiles las mujeres tienen mayor presencia que los hombres (57,8 y 42,2 por ciento, respectivamente). 
en la encuesta Casen. Vale destacar que la ESI incluso encuentra una menor participación de la mujer en el sector público. Esto se observa en el cuadro 4.

Cuadro 2. RELEVANCIA DEL SECTOR PÚBLICO EN EL MERCADO DEL TRABAJO, SEGÚN LA ESI

\begin{tabular}{lc}
\hline Año & Relevancia sector público (\%) \\
\hline 2010 & 5,15 \\
2011 & 5,72 \\
2012 & 5,80 \\
2013 & 5,48 \\
2014 & 5,70 \\
2015 & 5,57 \\
2016 & 5,41 \\
2017 & 5,64 \\
\hline
\end{tabular}

Fuente: Elaboración propia en base a ESI 2010-2017.

Cuadro 3. PARTICIPACIÓN LABORAL POR GÉNERO DENTRO DEL SECTOR PÚBLICO (CASEN)

\begin{tabular}{lcc}
\hline Año & Hombres (\%) & Mujeres (\%) \\
\hline 1990 & 73 & 27 \\
1992 & 77 & 23 \\
1994 & 74 & 26 \\
1996 & 74 & 26 \\
1998 & 68 & 32 \\
2000 & 71 & 29 \\
2003 & 69 & 31 \\
2006 & 66 & 34 \\
2009 & 59 & 41 \\
2011 & 59 & 41 \\
2013 & 56 & 44 \\
2015 & 57 & 43 \\
2017 & 58 & 42 \\
\hline
\end{tabular}

Fuente: Elaboración propia en base a encuestas Casen 1990-2017. 
Cuadro 4. PARTICIPACIÓN LABORAL POR GENERO DENTRO DEL SECTOR PÚBLICO (ESI)

\begin{tabular}{lcc}
\hline Año & Hombres (\%) & Mujeres (\%) \\
\hline 2010 & 59 & 41 \\
2011 & 61 & 39 \\
2012 & 59 & 41 \\
2013 & 62 & 38 \\
2014 & 60 & 40 \\
2015 & 60 & 40 \\
2016 & 61 & 39 \\
2017 & 59 & 41 \\
\hline
\end{tabular}

Fuente: Elaboración propia en base a ESI 2010-2017.

Otro factor que se debe tener en consideración es la evolución de los salarios públicos y privados. En promedio, los salarios públicos han sido, a excepción de 1992 y 2000, mayores que su contraparte privada. Esto se observa en el cuadro 5.

Cuadro 5. EVOLUCIÓN SALARIO PROMEDIO Y DESVIACIÓN ESTÁNDAR DEL SECTOR PÚBLICO Y PRIVADO (CASEN)

\begin{tabular}{lrrrr}
\hline Año & $\begin{array}{c}\text { Promedio } \\
\text { público }(\$)\end{array}$ & $\begin{array}{c}\text { Desv. estándar } \\
\text { público }(\$)\end{array}$ & $\begin{array}{c}\text { Promedio } \\
\text { privado }(\$)\end{array}$ & $\begin{array}{c}\text { Desv. estándar } \\
\text { privado (\$) }\end{array}$ \\
\hline 1990 & 88.620 & 81.536 & 81.506 & 161.806 \\
1992 & 123.017 & 111.549 & 125.132 & 259.154 \\
1994 & 210.671 & 221.514 & 168.831 & 840.483 \\
1996 & 230.303 & 217.154 & 214.446 & 401.741 \\
1998 & 279.618 & 287.483 & 262.269 & 560.880 \\
2000 & 275.088 & 235.104 & 285.536 & 574.913 \\
2003 & 348.638 & 310.598 & 304.104 & 728.016 \\
2006 & 373.890 & 369.670 & 306.914 & 531.303 \\
2009 & 529.820 & 509.446 & 407.978 & 680.343 \\
2011 & 568.844 & 823.004 & 432.335 & 654.257 \\
2013 & 600.801 & 592.472 & 415.137 & 530.919 \\
2015 & 682.734 & 597.452 & 455.820 & 520.617 \\
2017 & 758.109 & 673.612 & 514.022 & 605.741 \\
\hline
\end{tabular}

Fuente: Elaboración propia en base a encuestas Casen 1990-2017. 
Según datos de la ESI, dicha evolución también se ve reflejada. Se observa incluso que la brecha es mayor a la estimada vía encuesta Casen, lo que se puede observar en el cuadro 6.

Cuadro 6. EVOLUCIÓN SALARIO PROMEDIO Y DESVIACIÓN ESTÁNDAR DEL SECTOR PÚBLICO Y PRIVADO (ESI)

\begin{tabular}{lcccc}
\hline Año & $\begin{array}{c}\text { Promedio } \\
\text { público (\$) }\end{array}$ & $\begin{array}{c}\text { Desv. estándar } \\
\text { público }(\$)\end{array}$ & $\begin{array}{c}\text { Promedio } \\
\text { privado (\$) }\end{array}$ & $\begin{array}{c}\text { Desv. estándar } \\
\text { privado (\$) }\end{array}$ \\
\hline 2010 & 504.397 & 514.109 & 320.795 & 405.905 \\
2011 & 528.433 & 554.142 & 367.011 & 450.730 \\
2012 & 590.892 & 519.177 & 406.027 & 472.307 \\
2013 & 658.278 & 594.139 & 438.632 & 483.740 \\
2014 & 692.953 & 624.406 & 458.390 & 507.008 \\
2015 & 743.066 & 640.018 & 486.036 & 541.304 \\
2016 & 715.955 & 619.178 & 509.525 & 563.895 \\
2017 & 802.705 & 706.834 & 535.792 & 563.590 \\
\hline
\end{tabular}

Fuente: Elaboración propia en base a ESI 2010-2017.

\section{METODOLOGÍA}

En esta sección presentamos las distintas metodologías que aplicaremos en este estudio. Es muy relevante aclarar que todas ellas se realizan a nivel de cada base de datos independientemente, de forma que corresponden a estimaciones independientes de corte transversal.

\subsection{Premio del sector público}

Definiremos el premio del sector público como aquel porcentaje del salario del sector privado que un trabajador del sector público gana en promedio, tras controlar por observables. Este premio se calcula a través de una estimación lineal de la forma: ${ }^{11}$

${ }^{11}$ Ésta es la forma funcional exacta para la estimación con la encuesta Casen 2017. Estimaciones para otros años tienen menor cantidad de regresores debido a la menor cantidad de regiones o de niveles educacionales especificados. 


$$
\begin{aligned}
\log w_{k}=\beta_{0}+ & \beta_{1} \cdot P_{k}+\beta_{2} \cdot M_{k}+\beta_{3} \cdot \exp _{k}+\beta_{4} \cdot \exp _{k}^{2}+\sum_{i=1}^{12} \beta_{4+i} \cdot E d u c_{k, i} \\
& +\sum_{j=1}^{15} \beta_{16+j} \cdot R_{j}+u_{k}
\end{aligned}
$$

Aquí, $\log w_{k}$ corresponde al logaritmo del salario del trabajador $k$. La variable $P_{k}$ es una variable dicotómica que toma valor 1 si es que el sector del trabajador $k$ es público o 0 si es privado. La variable $M_{k}$ es una variable dicotómica que toma valor 1 si es que el trabajador $k$ es mujer o 0 si es hombre. La variable $\exp _{k}$ representa un proxy de experiencia laboral del trabajador $k$. Dicho proxy se calcula de la forma tradicional, correspondiente a la edad del individuo, menos su cantidad de años de escolaridad, menos seis años. El conjunto $\left\{E d u c_{k, i}\right\}_{i=1}^{12}$ comprende variables dicotómicas para los distintos niveles educacionales considerados. ${ }^{12}$ A su vez, el conjunto $\left\{R_{j}\right\}_{j=1}^{15}$ corresponde a variables dicotómicas que representan las regiones del país. ${ }^{13}$ El uso de dummies regionales se utiliza para controlar por la posibilidad de que las regiones se especialicen en distintos sectores productivos o que haya diferencias igualadoras entre ellas, y también son utilizadas en Cerda (2016).

Una vez planteado todo esto, se puede estimar vía mínimos cuadrados ordinarios el parámetro $\hat{\beta}_{1}$, que corresponde al premio del sector

${ }^{12}$ Los niveles considerados son: enseñanza básica, enseñanza media (técnicoprofesional y científico-humanista), educación técnica, educación universitaria y estudios de postgrado. Además, se precisa si dichos niveles están completos o incompletos. El nivel de postgrado se incorpora en 1994, sólo indicando si se tiene estudios de postgrado ya sean incompletos o no. En ese caso se usa una dummy que identifique dicho nivel, de forma que se controla por esa variable. A partir de 2013 existe la descomposición entre postgrado incompleto y completo, de forma que ahí se controla por ambas. La dummy omitida corresponde a todos aquellos que tienen un nivel educacional menor a enseñanza básica.

${ }^{13}$ Entre 1990 y 2006, había 13 regiones en el país. A partir de 2007 se incrementaron a 15 regiones, y a partir de septiembre de 2018, a 16 regiones. Dado que la Casen se adelanta a las implementaciones administrativas, las estimaciones se hacen con la máxima cantidad de regiones detalladas por esta encuesta en cada año. Esto permite que en 2006 la estimación ya considere 15 regiones, y que en 2017 considere 16 regiones. La justificación para esto es que los criterios para determinar qué es parte de una región y qué no son relevantes como control en este aspecto. La región omitida corresponde a la I, Región (de Tarapacá). 
público. Dado que la estimación es sobre el logaritmo del salario, dicho parámetro corresponde a un porcentaje del salario base, que en este caso es el salario privado.

\subsection{Premio del sector público por percentiles}

El premio anteriormente calculado corresponde a una estimación promedio del sector público. Como es lógico, dicho premio reúne a una gran cantidad de trabajadores distintos, que realizan diversas tareas y poseen diferentes características. Esta amplia heterogeneidad genera que la interpretación del premio sea compleja de realizar. Con la intención de medir un premio más específico a grupos se plantea realizar regresiones por cuantiles de ingreso.

Las regresiones por cuantiles son estimaciones no lineales que ponderan los datos de una muestra de forma distinta, con el propósito de enfocarse en un cuantil (percentil) en particular. La alternativa a este tipo de estimaciones es realizar una regresión lineal por mínimos cuadrados ordinarios, sólo para los datos de un percentil determinado. Sin embargo, acotar la muestra a niveles de deciles o de percentiles genera un doble problema para la regresión lineal: por un lado, se reduce sustancialmente la muestra, ${ }^{14} \mathrm{y}$, por otro, seleccionar partes determinadas de una distribución elimina la normalidad de la muestra, lo que perjudica la robustez de la estimación. En contraposición a esta aproximación, las regresiones por cuantiles, al ponderar todos los datos de la muestra, no pierden datos y es una estimación neutra frente a la distribución de la muestra, de forma que la pérdida de normalidad no la afecta.

Dado un cuantil determinado $\theta \epsilon(0,1)$, realizar una regresión de cuantiles implica resolver el siguiente problema:

$$
\hat{\beta}_{\theta}=\arg \min \left[\sum_{i: y \geq x_{i}^{\prime} \beta} \theta\left|y_{i}-x_{i}^{\prime} \beta\right|+\sum_{i: y \leq x_{i}^{\prime} \beta}(1-\theta)\left|y_{i}-x_{i}^{\prime} \beta\right|\right]
$$

Donde el parámetro $y_{i}$ se estima a través de:

$$
y_{i}=x_{i}^{\prime} \beta+u_{\theta}
$$

${ }^{14}$ Realizar una estimación para un percentil en particular implica perder el 99 por ciento de la muestra. 
Y donde se debe cumplir que:

$$
E\left(y_{i} \mid x_{i}\right)_{\text {Dado cuantil } \theta}=x_{i}^{\prime} \beta
$$

Es necesario mencionar que la estimación que realizaremos en (3) será con la misma forma funcional que usamos en (1), de forma que dichos resultados sean consistentes entre sí.

Lo anterior se puede escribir como un problema de programación lineal y ser resuelto vía métodos basados en simplex (Koenker y D’Orey 1987). De cada estimación, es decir para cada cuantil, se obtendrá un conjunto de estimadores $\hat{\beta}_{\theta}$, que son válidos sólo para dicho espacio de la distribución. De esta forma, tendremos estimadores $\hat{\beta}_{\theta}$ para caracterizar potencialmente toda la distribución condicional. Estos estimadores, ahora sí, permiten inferir cómo la política de salarios va influyendo a través de grupos de trabajadores relativamente homogéneos; al menos, en ingresos.

Para efectos de nuestras estimaciones consideraremos los percentiles $10,20,30,40,50,60,70,80,90$ y 99 de la distribución de salarios.

\subsection{Descomposición de Blinder-Oaxaca}

A modo de tener una estimación alternativa del premio promedio del sector público y darle robustez a los resultados, se realizó una descomposición de Blinder-Oaxaca. Esta descomposición consiste en separar las diferencias promedio (en este caso de salarios) en un componente explicado por características, y otro no explicado por características (que vendría a ser el premio). En particular, nos interesa saber qué medida no es explicada por características, y su magnitud dentro del total de la diferencia promedio. El objetivo es el mismo de la estimación anterior, por lo que representa una nueva estimación que nos permite chequear si los resultados son coherentes con los anteriores.

Esta metodología, aplicada a nuestro caso, consiste en estimar la siguiente ecuación tanto para el sector público como para el sector privado:

$$
\begin{gathered}
\log w_{k}=\beta_{0}+\beta_{1} \cdot M_{k}+\beta_{2} \cdot \exp _{k}+\beta_{3} \cdot \exp _{k}^{2}+\sum_{i=1}^{12} \beta_{3+i} \cdot E d u c_{k, i}+\sum_{j=1}^{15} \beta_{15+j} \cdot R_{j} \\
+u_{k}
\end{gathered}
$$


De forma que tendremos dos vectores de estimadores: los $\hat{\beta}$ correspondientes a la estimación del sector privado $\left(\hat{\beta}_{\text {priv }}\right)$ y los $\hat{\beta}$ correspondientes a la estimación del sector público $\left(\hat{\beta}_{p u b}\right)$. Si adicionalmente consideramos $\bar{X}_{\text {priv }}$ que es un vector con el promedio de las características del sector privado, y $\bar{X}_{p u b}$ su homólogo del sector público, tenemos entonces que la descomposición en dos partes de Blinder-Oaxaca ${ }^{15}$ se puede determinar como:

$D \equiv \log \bar{w}_{\text {pub }}-\log \bar{w}_{\text {priv }}=\left[\bar{X}_{\text {pub }}-\bar{X}_{\text {priv }}\right]^{\prime} \hat{\beta}_{\text {priv }}+\bar{X}_{\text {priv }}\left[\hat{\beta}_{\text {pub }}-\hat{\beta}_{\text {priv }}\right]$

Donde el primer sumando corresponde a la diferencia explicada por las distintas dotaciones promedio de los trabajadores de ambos sectores. El segundo sumando corresponde a la diferencia explicada por las valoraciones distintas de las características entre ambos sectores.

Para efectos de este artículo, consideramos que el premio del sector público va a venir dado por la magnitud del segundo sumando, ya que el sector público debiese valorar de igual manera — en teoría — las características de los trabajadores que el sector privado.

Adicionalmente, la proporción que nos interesa corresponde a la fracción de la diferencia total que no es explicada por las características de los individuos (que denominaremos $D_{N E}$ ), lo que corresponde a la siguiente expresión:

$$
D_{N E}=\frac{\bar{X}_{\text {priv }}\left[\hat{\beta}_{p u b}-\hat{\beta}_{\text {priv }}\right]}{\left(\log \bar{w}_{p u b}-\log \bar{w}_{\text {priv }}\right)}
$$

\subsection{Incentivos del sector público}

Prácticamente cualquier política de salarios que se tenga en el sector público generará diferencias con el sector privado, de forma que aparecerán incentivos a trabajadores de determinadas características a buscar empleo en un sector $\mathrm{u}$ otro.

\footnotetext{
${ }^{15}$ En inglés se denomina: Two-fold decomposition.
} 
Dado esto, queremos desarrollar una metodología que permita ver si hay incentivos para que una persona de determinadas características busque empleo en el sector público, independientemente de en qué sector trabaje actualmente. Consideraremos que el principal mecanismo de incentivo se produce a través de la paga de un mayor salario. ${ }^{16}$ De esta forma, diremos que una persona estará incentivada a buscar empleo en el sector público si es que existe un empleo en dicho sector que le pague un salario mayor al que actualmente tiene.

Para determinar ello, lo primero que estimaremos son las ecuaciones de salarios para los dos sectores de la economía (público y privado) por separado. Esto consiste en estimar la ecuación (1) para cada uno de los sectores:

$$
\begin{aligned}
\left(\log w \mid P_{k}=1\right) & \\
= & \beta_{0}+\beta_{1} \cdot M_{k}+\beta_{2} \cdot \exp _{k}+\beta_{3} \cdot \exp _{k}^{2}+\sum_{i=1}^{12} \beta_{4+i} \cdot E d u c_{k, i} \\
& +\sum_{j=1}^{15} \beta_{15+j} \cdot R_{j}+u_{k}
\end{aligned}
$$

$\left(\log w \mid P_{k}=0\right)$

$$
\begin{aligned}
& =\gamma_{0}+\gamma_{1} \cdot M_{k}+\gamma_{2} \cdot \exp _{k}+\gamma_{3} \cdot \exp _{k}^{2}+\sum_{i=1}^{12} \gamma_{3+i} \cdot E d u c_{k, i}+\sum_{j=1}^{15} \gamma_{15+j} \cdot R_{j} \\
& +u_{k}
\end{aligned}
$$

Donde se obtendrá un conjunto de estimadores $\hat{\beta}$ y estimadores $\hat{\gamma}$, que representan la valoración promedio de estos atributos en cada uno de los dos sectores.

Luego, un trabajador tendrá incentivos para buscar empleo en el sector público si el salario estimado de ese sector (dadas sus características) es mayor que el salario estimado para el sector privado; vale decir, el agente $k$ cumple con:

${ }^{16}$ Esto ignora, al igual que expresan Adamchik y Bedi (2000), los beneficios laborales no monetarios, sobre los que no hay cómo controlar. 


$$
\begin{gathered}
\left(\log \widehat{w} \mid P_{k}=1\right)>\left(\log \widehat{w} \mid D_{k}=0\right) \\
\left(\log \widehat{w} \mid P_{k}=1\right)-\left(\log \widehat{w} \mid D_{k}=0\right)>0
\end{gathered}
$$

Luego, se genera una variable dicotómica que tomará valor 1 si es que el trabajador tiene incentivos para buscar trabajo en el sector público y 0 si es que no los tiene, vale decir:

$$
q_{i}=\left\{\begin{array}{l}
1 \text { si }\left[\left(\log \widehat{w} \mid P_{k}=1\right)-\left(\log \widehat{w} \mid D_{k}=0\right)\right]>0 \\
0 \text { si }\left[\left(\log \widehat{w} \mid P_{k}=1\right)-\left(\log \widehat{w} \mid D_{k}=0\right)\right] \leq 0
\end{array}\right.
$$

Con esta variable tenemos un modelo probit, en el que, a través de una estimación de máxima verosimilitud, pretendemos encontrar los parámetros $\hat{\delta}$ que maximizan la expresión dada por:

$$
L=\prod_{i=1}^{N} \Phi\left(x_{i}^{\prime} \delta\right)^{q_{i}} \cdot\left[1-\Phi\left(x_{i}^{\prime} \delta\right)\right]^{1-q_{i}}
$$

Cabe destacar que la expresión $x_{i}^{\prime} \delta$ de (8) no puede contener las mismas variables explicativas que las ecuaciones de salarios de (6) y (7), dado que habrá problemas de identificación. Para evitar este problema, seguiremos los mismos pasos de Adamchik y Bedi (2000) y en la ecuación (8) utilizaremos la edad en vez de la experiencia como variable explicativa, y los años de escolaridad en vez de las dummies educacionales. La idea aquí es que, mientras la experiencia puede ser relevante a la hora de determinar un salario, la edad es relevante a la hora de ver dónde trabajar. Según los autores, esto se debe a que alguien joven tiene más facilidades para trabajar en el sector privado que alguien de mayor edad, quien, a su vez, podría preferir el sector público, dada su mayor estabilidad. El cambio en las variables de educación se debe a que agregar otro conjunto de variables dummies interactivas con el sector público podría generar problemas de colinealidad en la estimación. Adicionalmente, incluir la variable escolaridad permite obtener una visión promedio del incentivo a la educación. Las demás variables explicativas se mantendrán de la misma forma. Por último, vale mencionar que continuaremos controlando por región geográfica de Chile. 
De esta forma, estimaremos un modelo probit en el que las variables de interés que tendremos serán el género, la edad y los años de escolaridad.

Una vez que tengamos los coeficientes del modelo, estimaremos la probabilidad de que para cada persona exista un trabajo mejor remunerado en el sector público. Luego calcularemos la probabilidad promedio de que exista un trabajo mejor remunerado en el sector público para distintos grupos de personas: por género, por grupos de edad (20,30, $40 \mathrm{y}$ 50 años), y por grupos educacionales (enseñanza básica, media, técnica y universitaria completas).

\section{RESULTADOS}

En esta sección presentamos los resultados de las estimaciones para los diversos modelos planteados anteriormente. También comentamos los resultados más importantes, y las formas en que éstos deben ser interpretados. Los cuadros con los diversos resultados secundarios se encuentran en el Anexo.

\subsection{Premio del sector público}

La figura 1 muestra la evolución del premio para el sector público desde 1990 a 2017. Debemos recordar que este premio representa aquel porcentaje adicional al salario del empleado privado que, en promedio, obtiene un empleado público, tras controlar por características observables. Lo más notorio de este gráfico es que ha habido un importante aumento del premio salarial de los empleados públicos en el último cuarto de siglo. Mientras a principios de la década de 1990 fluctuaba entre menos 5 por ciento (es decir un castigo más que un premio) y 5 por ciento, en el último año de la encuesta llegó a 20 por ciento, con una clara tendencia ascendente en el período.

A partir de 1998 el premio sólo cae en una ocasión, que corresponde al periodo entre 2009 y 2011, cuando pasó de 12,87 por ciento a 12,15 por ciento; es decir, un cambio muy menor.

El cuadro 7 muestra la evolución y significancia de los índices estimados. Se puede observar que para los años 1990, 1994, 1996 y 1998 no es posible rechazar la hipótesis nula (al 90 por ciento de confianza); es decir, de que el premio público sea cero por ciento. En el año 1992 
Figura 1. EVOLUCIÓN DEL PREMIO DEL SECTOR PÚBLICO PARA EL PERIODO 19902017 (CASEN)

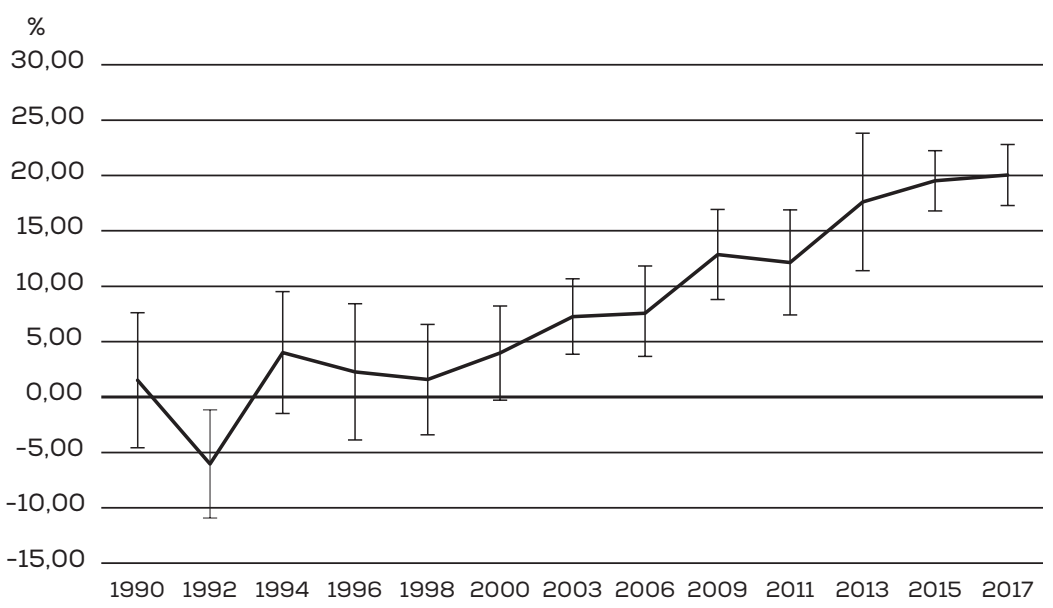

Nota: Intervalos de confianza al 95 por ciento. Detalles en Anexo.

Cuadro 7. COEFICIENTES DEL PREMIO PÚBLICO Y SIGNIFICANCIA ESTADÍSTICA (CASEN)

\begin{tabular}{lcccc}
\hline Año & Premio & Significancia & $\mathrm{N}$ & $\mathrm{R}^{2}$ \\
\hline 1990 & 1,51 & & 32944 & 0,33 \\
1992 & $-6,05$ & $* *$ & 46370 & 0,34 \\
1994 & 4,01 & & 56399 & 0,38 \\
1996 & 2,27 & & 43003 & 0,39 \\
1998 & 1,57 & & 60565 & 0,43 \\
2000 & 3,97 & $*$ & 72459 & 0,43 \\
2003 & 7,27 & $* * *$ & 81867 & 0,41 \\
2006 & 7,57 & $* * *$ & 90014 & 0,38 \\
2009 & 12,87 & $* * *$ & 80890 & 0,41 \\
2011 & 12,15 & $* * *$ & 71835 & 0,39 \\
2013 & 17,92 & $* * *$ & 63544 & 0,44 \\
2015 & 19,52 & $* * *$ & 73148 & 0,44 \\
2017 & 20,04 & $* * *$ & 58795 & 0,44 \\
\hline
\end{tabular}

Nota: * indica una significancia al 90 por ciento, ${ }^{* \star}$ al 95 por ciento $y$ ** al 99 por ciento. 
existió un castigo para el sector público de seis por ciento, lo que se debe a un alza importante de salarios del sector privado mayoritariamente, más que a una disminución en los salarios del sector público.

Por otro lado, el crecimiento del premio entre 1998 y 2017 es bastante pronunciado. El premio crece en ese periodo a un ritmo promedio de 0,97 puntos porcentuales por año. A partir de 2000 dicho premio es estadísticamente significativo (se rechaza que sea cero).

La figura 2 exhibe las estimaciones con la ESI. Como se aprecia, la ESI no reporta el aumento del premio que, según la encuesta Casen, ha presentado el sector público entre 2010 y 2017, pero sí confirma niveles de premio en torno al 20 por ciento. ${ }^{17}$ La figura 3 muestra la evolución del premio a partir de la encuesta Casen, y las estimaciones de la ESI para los años 2011, 2013, 2015 y 2017. Aunque no muy diferentes, el estimado con la encuesta Casen está por debajo del estimado con la ESI.

Figura 2. EVOLUCIÓN DEL PREMIO DEL SECTOR PÚBLICO PARA EL PERIODO 20102017 (ESI)

$\%$

30,00

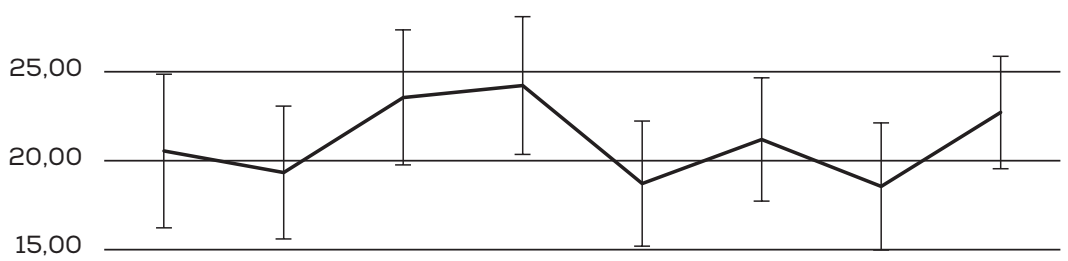

10,00

5,00

0,00

$\begin{array}{llllllll}2010 & 2011 & 2012 & 2013 & 2014 & 2015 & 2016 & 2017\end{array}$

Nota: Intervalos de confianza al 95 por ciento. Detalles en el Anexo.

Fuente: Elaboración propia.

${ }^{17}$ En las estimaciones con la ESI los premios son todos estadísticamente significativos al 99 por ciento. 
Figura 3. EVOLUCIÓN DEL PREMIO DEL SECTOR PÚBLICO PARA EL PERIODO 19902015 (CASEN MÁS DATOS ESI PARA 2011, 2013, 2015 Y 2017)

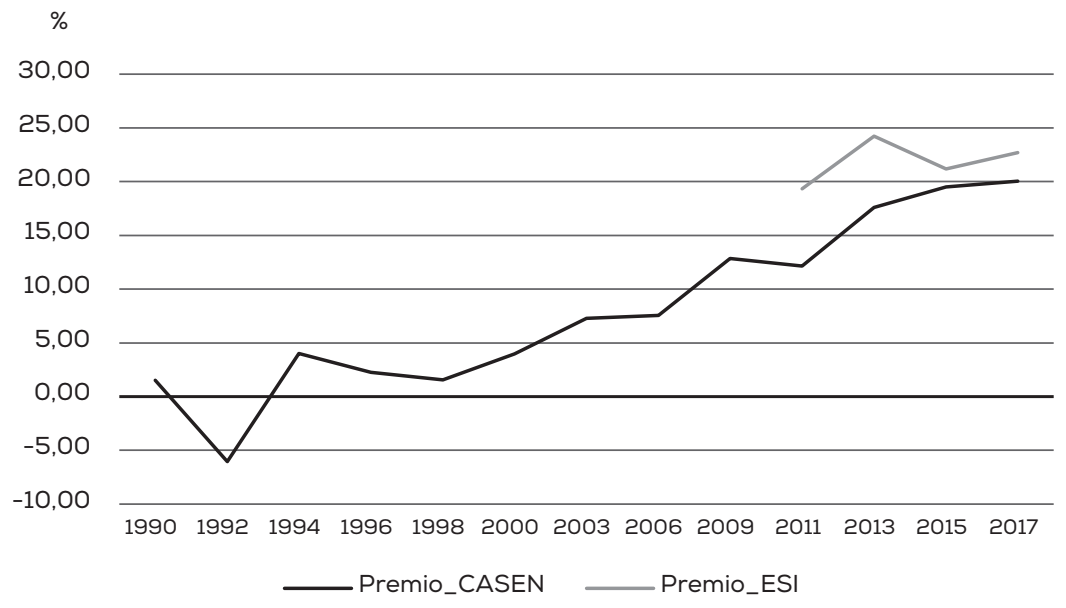

Fuente: Elaboración propia.

\subsection{Premio del Sector Público por percentiles}

Como ya habíamos comentado, el premio anteriormente expuesto es un premio promedio para el conjunto de la economía, por lo que resulta interesante ver cómo este premio se comporta a través de distintos cuantiles de ingreso.

Los cuantiles (percentiles) de ingreso utilizados fueron: 10 por ciento, 20 por ciento, 30 por ciento, 40 por ciento, 50 por ciento, 60 por ciento, 70 por ciento, 80 por ciento, 90 por ciento y 99 por ciento. La figura 4 representa la evolución de los premios para cada uno de estos cuantiles a través del tiempo.

Como podemos observar, los percentiles más bajos de la distribución consistentemente tienen un premio positivo que ronda entre el 10 por ciento y 20 por ciento. El movimiento de los percentiles más altos es mucho más dinámico, y vemos que suben paulatinamente a lo largo de la serie. El caso más evidente de esta situación es la evolución del percentil 99, que pasa de tener un castigo público cercano al 50 por ciento a terminar con un premio de 14 por ciento.

Esta evolución de la política salarial chilena llama la atención en algunos aspectos. En primer lugar, existe una cierta percepción de que 
Figura 4. EVOLUCIÓN PREMIO DEL SECTOR PÚBLICO POR PERCENTILES (CASEN)

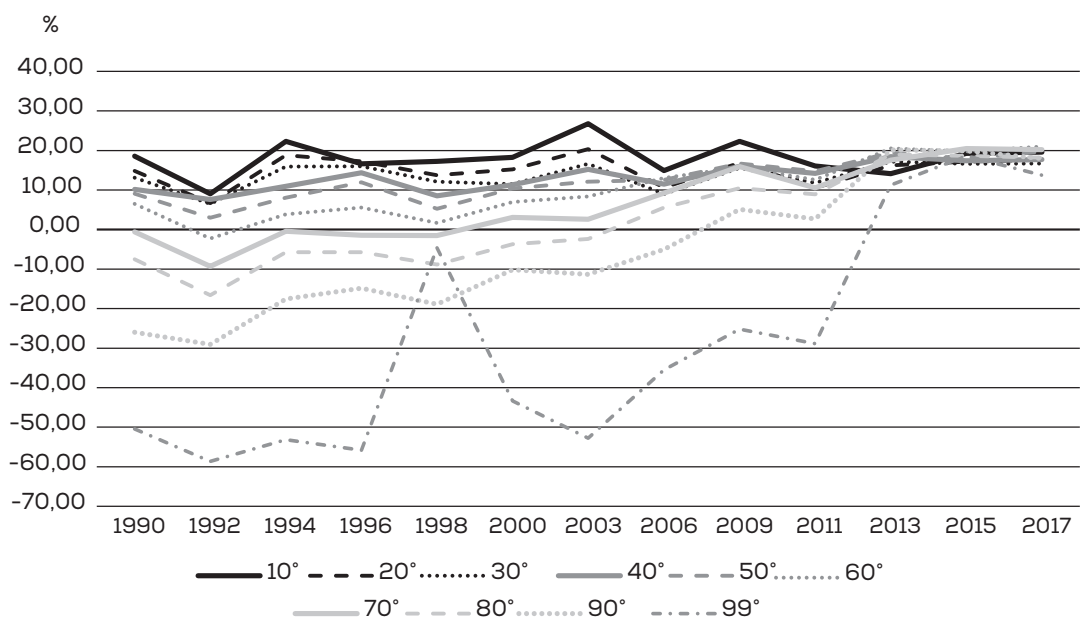

Fuente: Elaboración propia.

los trabajadores menos calificados son más favorecidos por trabajar en el sector público. Lo que nuestras estimaciones muestran es que esto era así hasta hace unos años atrás, pero que las diferencias por percentiles se han ido cerrando y que incluso en 2017 están prácticamente cerradas. Así, el premio para todos los percentiles es en torno a 18,5 por ciento, a excepción del percentil 99, en el que es 14 por ciento.

En segundo lugar, se podría argumentar que las negociaciones salariales del sector público tienen como uno de sus objetivos reducir las desigualdades de ingresos en el sector público. Si ése fuera el caso, entonces los premios debieran bajar a medida que aumenta el ingreso. Vemos que eso fue así históricamente, pero que ha ido perdiendo fuerza en el tiempo. En síntesis, lo novedoso de estas estimaciones es ver cómo se ha ido cerrando la diferencia de premio en el tiempo, para llegar a ser en la actualidad muy similar, independientemente del percentil de ingreso.

Por su parte, las estimaciones con datos de la ESI sostienen parcialmente las estimaciones realizadas vía encuesta Casen, lo que se puede observar en la figura 5. Esto, ya que confirman una convergencia hacia un premio mayor a 20 por ciento, aunque muestran que los percentiles 90 y 99 están por debajo de eso, si bien sobre 15 por ciento. 
Figura 5. EVOLUCIÓN PREMIO DEL SECTOR PÚBLICO POR PERCENTILES (ESI)

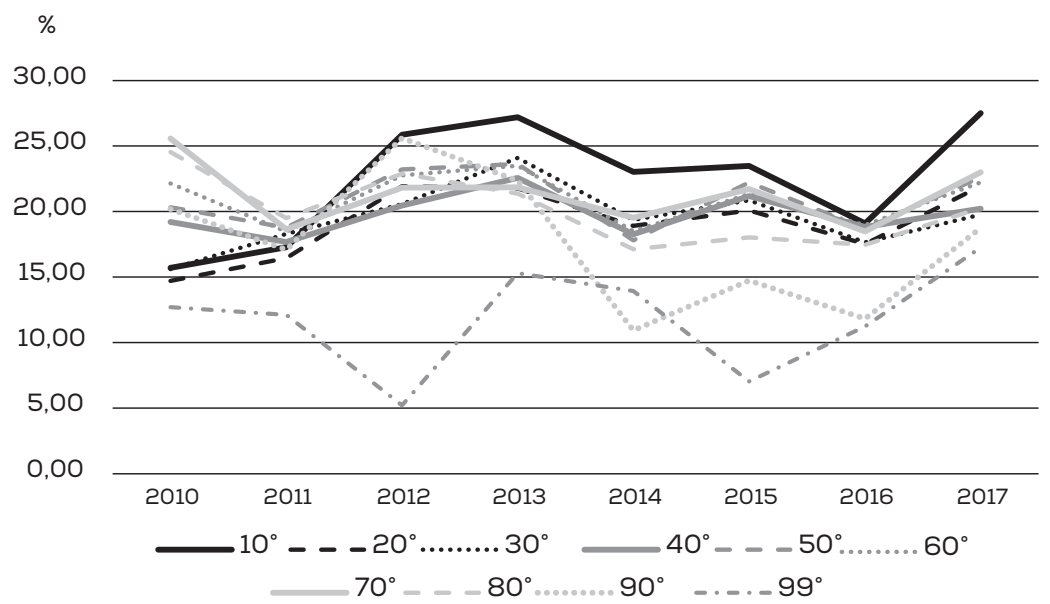

Fuente: Elaboración propia.

\subsection{Descomposición de Blinder-Oaxaca}

Una forma adicional de estimar el premio del salario de los empleados públicos controlando por características es mediante la denominada descomposición de Blinder-Oaxaca. Esto se realiza con la intención de obtener una robustez a nuestras estimaciones. En el cuadro 8 se muestran los resultados. Como era de esperar, ambas estimaciones, la anterior mediante la estimación lineal del parámetro asociado a una variable dummy por año y ésta - Blinder-Oaxaca - arrojan resultados similares. Las diferencias del premio entre ambas estimaciones nunca superan el 1 por ciento. ${ }^{18}$

Por su parte, la figura 6 muestra la evolución de la proporción de la diferencia promedio de salarios no explicada por características observables. Esto equivale a ver qué porcentaje de la diferencia promedio de salarios corresponde al premio. Como se puede ver, desde 1998 dicha proporción ha aumentado consistentemente a lo largo de los distintos periodos. Cabe hacer notar que, en un comienzo de la década de 1990, la proporción del premio no explicada por características era menor al

\footnotetext{
${ }^{18}$ Lo mismo ocurre cuando se hacen las estimaciones con la ESI.
} 
Cuadro 8. EVOLUCIÓN DEL PREMIO DEL SECTOR PÚBLICO VÍA REGRESIÓN CON DUMMY Y BLINDER-OAXACA (CASEN)

\begin{tabular}{lcc}
\hline Año & Dummy & Blinder-Oaxaca \\
\hline 1990 & 1,51 & 1,54 \\
1992 & $-6,05$ & $-5,93$ \\
1994 & 4,01 & 4,08 \\
1996 & 2,27 & 2,32 \\
1998 & 1,57 & 1,59 \\
2000 & 3,97 & 4,04 \\
2003 & 7,27 & 7,26 \\
2006 & 7,57 & 7,35 \\
2009 & 12,87 & 12,92 \\
2011 & 12,15 & 12,21 \\
2013 & 17,91 & 18,07 \\
2015 & 19,52 & 19,61 \\
2017 & 20,04 & 20,07 \\
\hline
\end{tabular}

Fuente: Elaboración propia.

Figura 6. EVOLUCIÓN PROPORCIÓN DEL PREMIO NO EXPLICADA POR CARACTERÍSTICAS OBSERVABLES (CASEN)

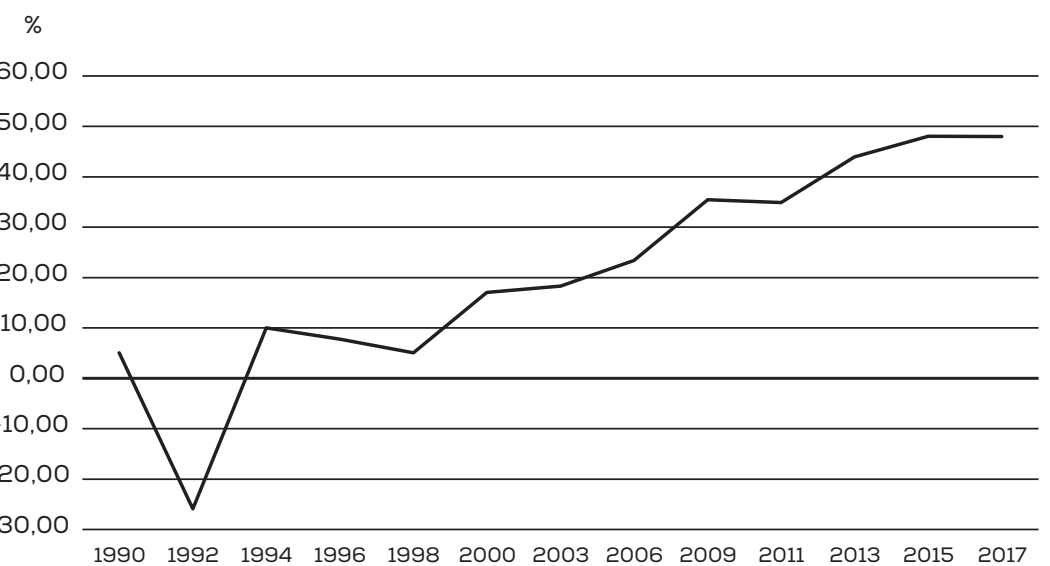

Fuente: Elaboración propia. 
10 por ciento. Sin embargo, esta situación cambió significativamente, llegando a la actualidad a cerca del 50 por ciento. ${ }^{19}$

\subsection{Incentivos del sector público}

En esta sección veremos cómo las características de los trabajadores afectan la probabilidad de que existan, para cada uno de ellos, trabajos mejor remunerados en el sector público que en el privado.

Los componentes presentados a continuación representan la probabilidad promedio de que un agente agrupado en una característica encuentre un empleo mejor renumerado en el sector público. Esto último no implica que dicho individuo gane el empleo, sino que el puesto exista. Las características sobre las que mediremos las probabilidades son: género, edad y nivel educacional completo.

Con respecto al género, se ve que el sector privado genera mayores incentivos a las mujeres que a los hombres a lo largo de toda la serie, algo que se puede apreciar en la figura 7. Si bien la serie de las mujeres presenta más movimientos que la de los hombres, la primera siempre está por sobre la segunda. En términos de valores, vemos que en 1990 las mujeres tenían un 86 por ciento de probabilidad de ser mejor pagadas en el sector público, mientras que para los hombres este valor era de 39 por ciento. Hacia 2017, las mujeres tenían un 91 por ciento de probabilidad de tener un mejor salario en el sector público, mientras que en los hombres dicha cifra crece a 82 por ciento.

En los resultados de la ESI encontramos algo similar, lo que puede verse en la figura 8. Si bien no se detecta el nivel de brecha que detectamos en la encuesta Casen, sí se mantiene el incentivo a las mujeres por sobre los hombres. En términos de valores, en 2017 las mujeres tenían 89 por ciento de probabilidad de que existiera un trabajo mejor remunerado en el sector público, mientras que para los hombres dicha probabilidad era de 82 por ciento.

Sobre la edad, vemos que tanto al comienzo como al final de la serie los incentivos a las edades son parejos, aunque aumentan en el tiempo, lo que se puede observar en la figura 9. En 1990, a cualquier edad se tenía una probabilidad del 64 por ciento de que existiera un

${ }^{19} \mathrm{Al}$ estimar con datos de la ESI, la proporción pasa de 52 por ciento en 2010 a 53 por ciento en 2017 . 
Figura 7. EVOLUCIÓN INCENTIVO A MUJERES (CASEN)

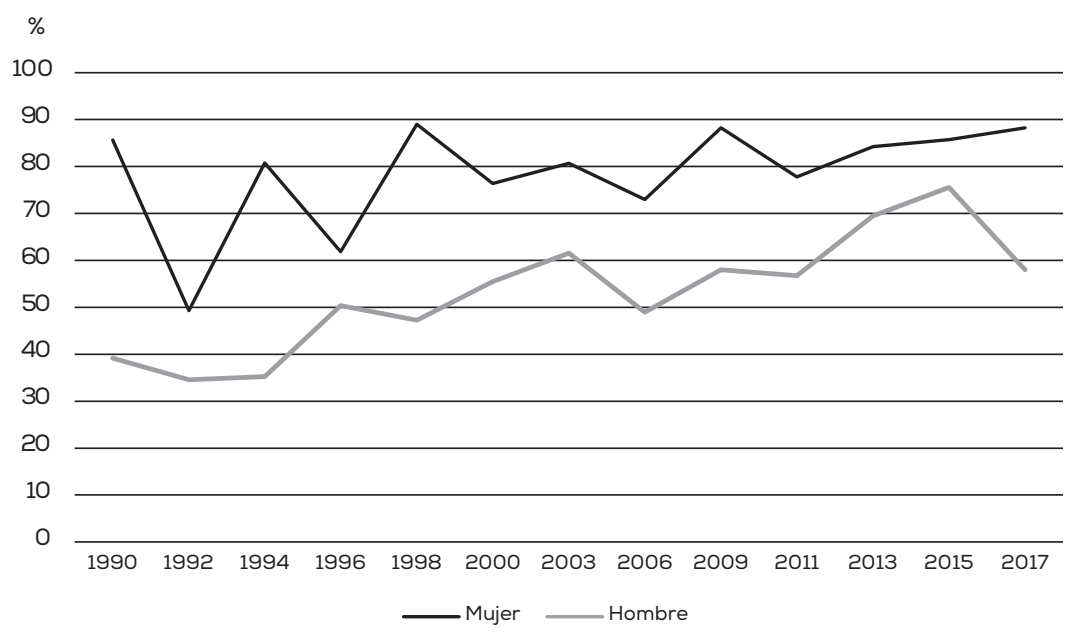

Nota: Dada la escala del gráfico, los intervalos de confianza son muy pequeños para ser reportados. Diferencias estadisticamente significativas al 99 por ciento en todos los años.

Fuente: Elaboración propia.

Figura 8. EVOLUCIÓN INCENTIVO A MUJERES (ESI)

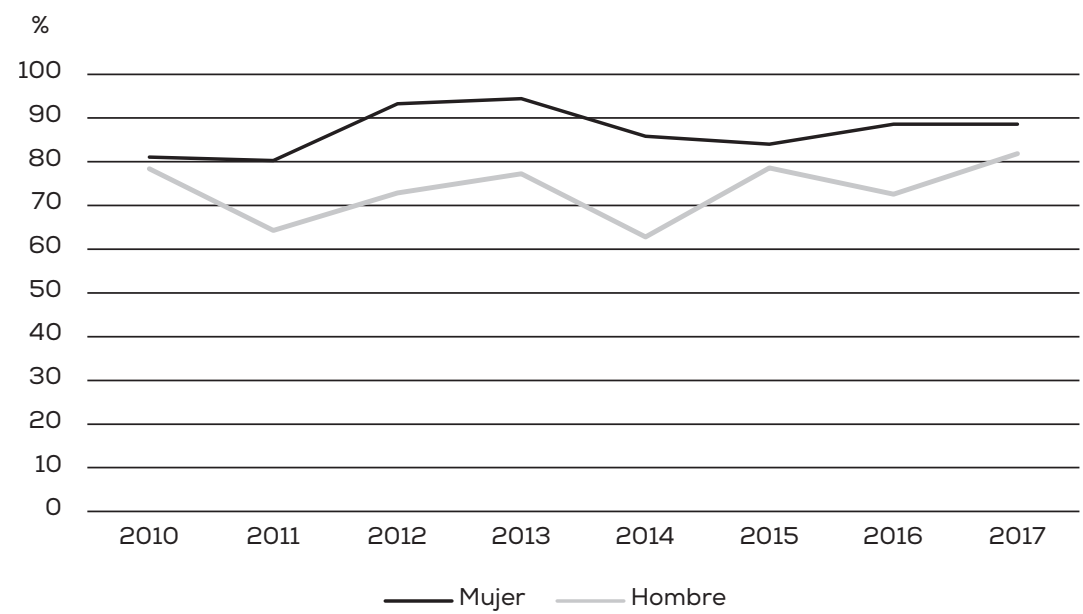

Nota: Dada la escala del gráfico, los intervalos de confianza son muy pequeños para ser reportados. Diferencias estadisticamente significativas al 99 por ciento en todos los años.

Fuente: Elaboración propia. 
Figura 9. EVOLUCIÓN INCENTIVO A LA EDAD (CASEN)

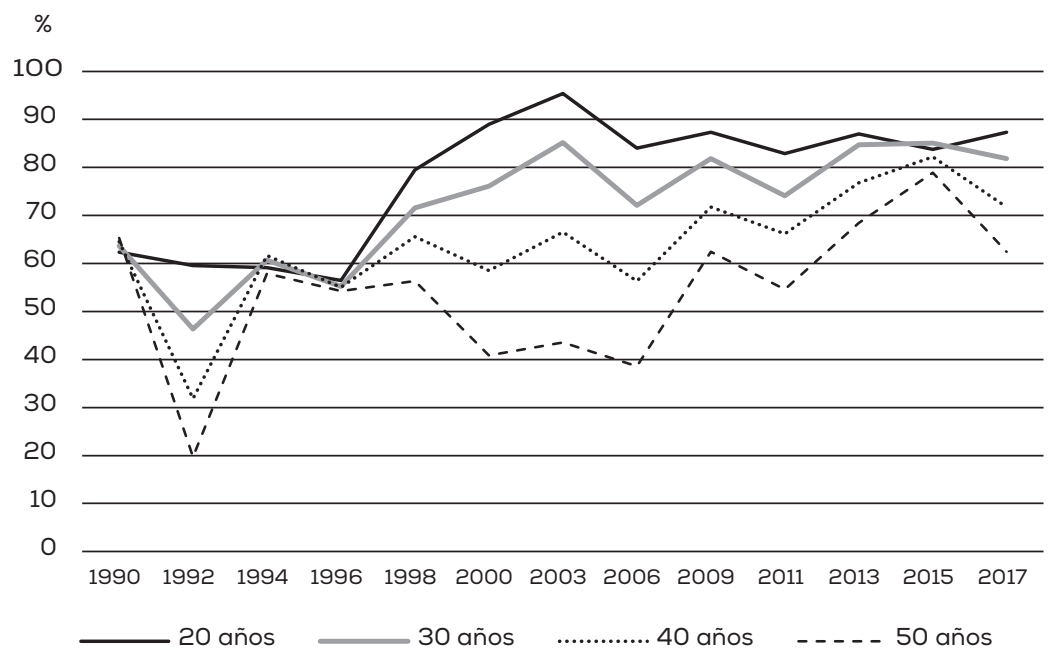

Nota: Dada la escala del gráfico, los intervalos de confianza son muy pequeños para ser reportados.*

Fuente: Elaboración propia.

trabajo mejor remunerado en el sector público. Dicha probabilidad se dispersa en 2017, para un rango entre el 83 y el 90 por ciento. Sin embargo, lo más interesante se da en la mitad de la serie, entre los años 1998 y 2011, donde se aprecian claros incentivos a los más jóvenes. En 2003, a los 20 años, la probabilidad de que existiera un trabajo mejor remunerado en el sector público era de 95 por ciento; a los 30 años, de 85 por ciento; a los 40 años, de 67 por ciento, y a los 50 años, sólo era de 44 por ciento. ${ }^{20}$

En cuanto a los incentivos por niveles educacionales completos, los resultados se observan en la figura 10. En 1990, para todos los niveles educacionales la probabilidad de que existiera un trabajo mejor remunerado en el sector público era en torno al 60 por ciento. Hacia 2017 dichos valores se vuelven heterogéneos por nivel educacional. Las

* Diferencias estadísticamente significativas al 99 por ciento en los cuatro grupos en 1992, 1998, 2000, 2003, 2006, 2009, 2011 y 2017.

${ }^{20}$ En las estimaciones utilizando la ESI, los resultados son coherentes. En 2010 la probabilidad de encontrar trabajos mejor remunerados en el sector público era mayor para los más jóvenes. Al igual que la estimación usando la encuesta Casen, se observa que dichas brechas se cierran hacia 2017, aunque no completamente. 
Figura 10. EVOLUCIÓN INCENTIVO A LA ESCOLARIDAD (CASEN)

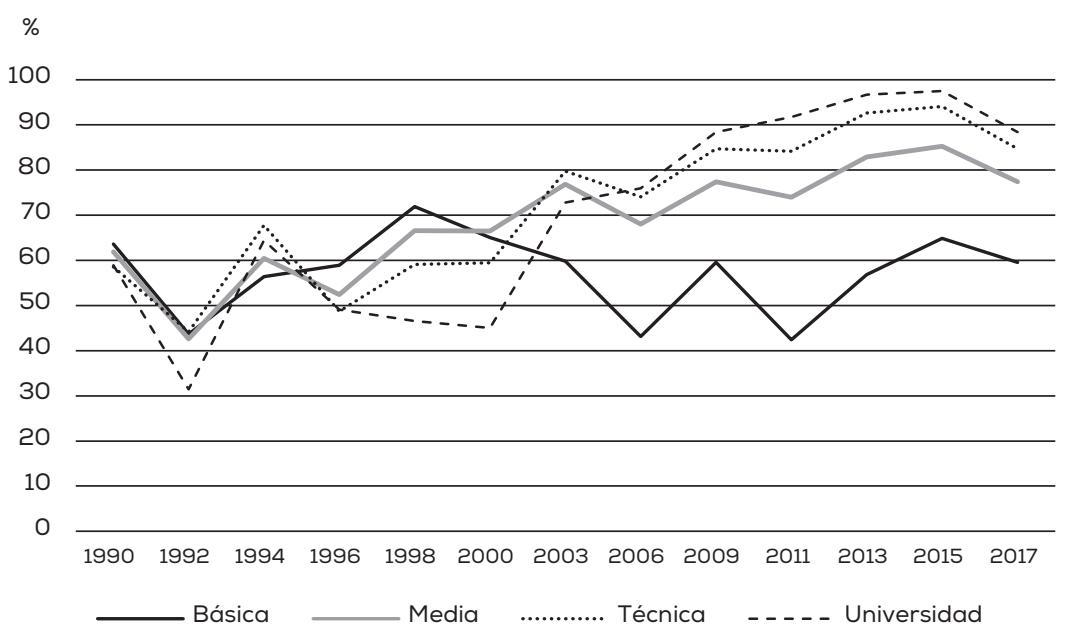

Nota: Dada la escala del gráfico, los intervalos de confianza son muy pequeños para ser reportados.*

Fuente: Elaboración propia.

probabilidades son 73 por ciento, 89 por ciento, 95 por ciento y 98 por ciento, para educación básica, educación media, educación técnica y educación universitaria, respectivamente. ${ }^{21}$

\section{DISCUSIÓN}

Los resultados expuestos en la sección anterior son llamativos, y requieren de una discusión que ayude a elaborar algunas hipótesis sobre los factores que los pueden estar generando. En primer lugar, es prudente mencionar algunos aspectos econométricos de las estimaciones. Se debe recordar que en éstas hay un conjunto de no observables que pueden estar influyendo en los resultados, en particular factores psicológicos que afecten a los trabajadores y a su productividad. Así,

* Diferencias estadísticamente significativas al 99 por ciento en los cuatro grupos en 1994, 1998, 2000, 2003, 2009, 2011, 2013, 2015 y 2017.

${ }^{21}$ En este caso los resultados utilizando los datos de la ESI son algo distintos. Muestran cifras sobre 90 por ciento para todo el período (2010-2017) en educación profesional y técnica; sobre 80 por ciento en media, y entre 40 y 70 en básica. 
sólo por este hecho, una explicación potencial del premio encontrado en el sector público sería que los trabajadores de dicho sector poseen no observables que los hacen más productivos que los trabajadores del sector privado, y que dichos no observables son recompensados a través de mayores salarios. Lo anterior podría esperarse de un contexto de competencia perfecta, en el cual a los trabajadores se les paga el valor del producto marginal que genera el último trabajador contratado, y en el cual exista un sesgo de selección que hace que quienes trabajen en el sector público no sea un conjunto aleatorio de los trabajadores. Sin embargo, pensar que los empleados del sector público son 20 por ciento más productivos que sus pares del sector privado parece poco plausible. Por otro lado, si bien es posible que exista un sesgo de selección entre los trabajadores que se emplean en el sector público, no es evidente qué tipo de variables podrían modelar dicha decisión..$^{22}$ En este sentido, es preferible asumir dicho posible sesgo a intentar explicarlo de forma poco satisfactoria.

La ausencia de ciertas variables - denominadas no observables - es persistente en este tipo de estimaciones, ya que la encuesta Casen no incluye un cuestionario psicológico que permita obtenerlas. ${ }^{23}$ Por otra parte, utilizar efectos fijos en encuestas longitudinales, como la EPS, capturaría no sólo dichos factores, sino también los que sean comunes entre los distintos periodos, como por ejemplo el poder de negociación, que es importante mantener fuera de la estimación. De esta forma, no existe una forma aceptada en la literatura sobre cómo lidiar con los no observables.

Habiendo discutido aspectos econométricos que podrían ser parte de la explicación de la brecha, pasamos ahora a considerar posibles explicaciones económicas. Un efecto que puede estar jugando un rol relevante en el premio encontrado es el de la sindicalización. Esta última permite generar un mayor poder de negociación a la hora de establecer los salarios, el cual eventualmente permitiría conseguir rentas — esto es, pagos que van más allá del valor de la productividad marginal del último trabajador contratado-, que se estarían midiendo a través del

${ }^{22}$ Cuando se enfrenta un problema de sesgo de selección para medir la brecha de género, la literatura usualmente utiliza medidas como los años de escolaridad de la mujer y la presencia de niños menores de seis años en el hogar, lo que tiene sentido dado el problema. Sin embargo, no es claro qué variables inciden en las preferencias de trabajar en un sector $u$ otro.

${ }^{23} \mathrm{Y}$ carece de sentido, dada la finalidad de la encuesta, que es medir la pobreza. 
cálculo del premio salarial. La literatura económica es abundante en torno al estudio de los efectos de la participación en sindicatos sobre el efecto en los salarios. Robinson y Tomes (1984) muestran que el premio del sector público encontrado para Canadá estaría principalmente reflejando las consecuencias de una mayor sindicalización de dicho sector. Al momento de analizar por grupos de calificación, Card (1996) muestra, utilizando datos de Estados Unidos, que los sindicatos ayudan a subir las remuneraciones de los trabajadores de menor calificación, mientras que los de alta calificación pueden incluso quedar en una peor situación si ingresan al sindicato. Sin embargo, la evidencia sobre premio al salario y calificación es mixta. Así, por ejemplo, Blanchflower y Bryson (2010), quienes utilizan datos del Reino Unido, muestran que el premio de afiliación a sindicatos en el sector público es mayor para los trabajadores de pagos más altos, mientras que el premio de afiliación en el sector privado es mayor para los trabajadores de menores ingresos.

Teniendo esta literatura en consideración, es posible que el sector público tenga un premio con respecto al sector privado debido a una distinta naturaleza sindical entre ambos sectores, como plantean Robinson y Tomes (1984). Si esto fuese cierto, al momento de controlar por la sindicalización de los individuos podríamos observar que el premio del sector público debiese caer. ${ }^{24}$ Dado que en el sector público en Chile no se pueden formar sindicatos, ${ }^{25}$ cuando hablamos de sindicalización de este sector nos referimos al grado de centralización de la negociación por sus condiciones salariales, que notablemente en Chile es muy elevada. En efecto, sí están permitidas las asociaciones de funcionarios de los sectores que no tienen acceso a sindicalización, ${ }^{26}$ generando asociaciones como la ANEF, que reemplazan en alguna medida dicha función articuladora. Por otra parte, la sindicalización en el sector privado no supera el 20 por ciento.

${ }^{24}$ Esto es el premio del sector público libre del efecto de la sindicalización, ya que entonces el generador del premio sería la sindicalización existente y no el sector público en sí.

${ }^{25}$ El estatuto administrativo en Chile prohíbe: "Organizar o pertenecer a sindicatos en el ámbito de la Administración del Estado; dirigir, promover o participar en huelgas, interrupción o paralización de actividades, totales o parciales, en la retención indebida de personas o bienes, y en otros actos que perturben el normal funcionamiento de los órganos de la Administración del Estado".

${ }^{26}$ Ella se realiza en base a la Ley 19.296, que "Establece normas sobre asociaciones de funcionarios de la Administración del Estado". 
También existe literatura que mide el efecto de la especialización y la baja movilidad que implica trabajar en un solo sector (Antel 1986; Garloff y Kuckulenz 2006). Siguiendo esta línea, puede ser que el sector público esté pagando mayores salarios en un intento de compensar la especialización y baja movilidad que podría generar este sector. Lo anterior tiene sentido económico si trabajar en el sector público otorga una experiencia laboral que no es valorada en el resto de los sectores productivos (en este caso, el sector privado agregado). Si esto fuese así, para incentivar a los trabajadores a participar en el sector público habría que pagarles un premio, de modo de compensar la falta de movilidad sectorial que están aceptando al trabajar allí, la que sería creciente con el paso del tiempo, lo que podría implicar un premio cada vez mayor. ${ }^{27} \mathrm{Si}$ éste fuera el caso, debiera esperarse que el premio del sector público se vea reflejado a través de un mayor pago a la experiencia laboral en el cargo. ${ }^{28}$ Las estimaciones por Blinder-Oaxaca, sin embargo, no sugieren un pago distinto para la experiencia laboral potencial y su cuadrado entre los sectores público y privado. Pero esto no nos permite descartar esta hipótesis. La experiencia laboral potencial es la cantidad de años que una persona debiese poseer de experiencia laboral en caso de no tener lagunas laborales. Pese a esto, esa medida no implica que esos años de experiencia laboral hayan sido en el mismo cargo donde se encuentra trabajando ahora. De esta forma, uno podría controlar por este hecho al incluir una variable que indicase cuánto tiempo un trabajador ha estado en el mismo puesto. ${ }^{29}$ Sin embargo, su inclusión no es trivial, ya que su interpretación genera ruido. Si efectivamente el coeficiente da con el signo esperado y es significativo, no necesariamente se puede concluir que ése es el fenómeno que genera la brecha, ya que, en ausencia de una variable que mida la sindicalización, puede existir sesgo debido a variables omitidas. ${ }^{30}$

${ }^{27}$ Este "podría" es estrictamente tentativo, ya que, por una parte, trabajar en el sector público genera una inmovilidad al individuo que debiera compensarse, pero, por otra, en la medida en que pase el tiempo el costo alternativo en el sector privado es menor, lo que es una fuerza en contra que el premio suba con los años en el puesto.

${ }^{28}$ Lo que en inglés se denomina job tenure.

${ }^{29}$ Disponible en la encuesta Casen desde el año 2000.

${ }^{30}$ Es importante diferenciar el efecto de la experiencia en el cargo del efecto de la sindicalización. De lo contrario, si se realiza la estimación con la experiencia en el cargo, habrá un problema de variables omitidas, ya que el premio podría deberse a un esfuerzo por compensar una menor movilidad - que aumenta con los años en el cargo- o, bien, la relación podría tener un sentido inverso: se está más años en el cargo porque se recibe un premio producto del mayor poder de negociación del sindicato. 
Así, entonces, existe una serie de factores que pueden estar incidiendo en la generación del premio salarial que se encuentra en el sector público, y que es importante tener en consideración a la hora de evaluar distintas políticas públicas.

\section{CONCLUSIONES}

Este artículo presenta un análisis sobre la relación existente entre las remuneraciones en el sector público y el sector privado, con la particularidad de que se realizaron dichas estimaciones para un periodo particularmente largo, que corresponde desde 1990 a 2017. Dentro de los resultados más importantes a destacar, se encontró que el premio público ha aumentado significativamente en el país, pasando de 1,5 por ciento en 1990 a 20 por ciento en 2017. Esto significa que, controlando por características de las personas (experiencia, educación, edad, género, etcétera), un trabajador en el sector público gana 20 por ciento más que el mismo trabajador en el sector privado. A nivel de percentiles, se observó un cambio significativo, ya que mientras en 1990 el percentil 10 recibía 19 por ciento de premio y el percentil 90 recibía 26 por ciento de castigo. En 2017 el percentil 10 tiene un premio de 19,5 por ciento y el percentil 90, un premio de 18 por ciento. Es decir, en la década de 1990 los mayores ingresos no tenían un premio por trabajar en el sector público, e incluso en algunos casos tenían un castigo (ganaban menos que en el sector privado), mientras que los funcionarios de ingreso bajo tenían un premio alrededor de 20 por ciento. Con el tiempo el premio a los ingresos más bajos se ha mantenido más o menos constante, mientras que el premio a los mayores salarios ha aumentado y convergido en el tiempo a los primeros. Esto ayuda a concluir que el aumento del premio general se debió a un aumento del premio en los percentiles de mayores ingresos, mientras el premio en los percentiles de menores ingresos se mantuvo constante.

También se realizó una descomposición de Blinder-Oaxaca que, junto con reafirmar las estimaciones realizadas del premio, nos indica que éste es cada vez más relevante a la hora de explicar la diferencia entre salarios públicos y privados. Esto debido a que en 1990 el premio representaba el 5 por ciento de la diferencia promedio de salarios, mientras que en 2017 representaba el 48 por ciento. 
Con respecto a los incentivos que genera la política de remuneración del sector público, se ve que hay incentivos hacia las mujeres; que existió un incentivo a los jóvenes pero que ha ido desapareciendo, y que existe un constante incentivo a las personas con estudios técnicos y universitarios completos.

Por último, se analizó una serie de factores econométricos y económicos que pueden estar explicando las magnitudes y la evolución de los premios salariales en el sector público, los que deben ser considerados a la hora de elaborar políticas públicas en torno a este tema.

\section{REFERENCIAS}

Adamchik, Vera \& Arjun Bedi. 2000. "Wage differentials between the public and the private sectors: Evidence from an economy in transition". Labour Economics 7: 203-224.

Antel, John. 1986. "Human capital investment specialization and the wages effects of voluntary labor mobility". The Review of Economics and Statistics 68 (3): 477-483.

Balbontín, Rodrigo, Jorge Rodríguez \& Alex Seeman. 2018. "Principales tendencias del empleo en el gobierno central". Serie de Estudios de Finanzas Públicas. Dirección de Presupuestos, Gobierno de Chile. Febrero.

Blanchflower, David \& Alex Bryson. 2010. "The wage impact of trade unions in the UK public and private sectors". Economica 77: 92-109.

Blinder, Alan. 1973. "Wage discrimination: Reduced form and structural estimates". Journal of Human Resources 8 (4): 436-455.

Card, David. 1996. "The effect of unions on the structure of wages: A longitudinal analysis". Econometrica 64 (4): 957-979.

Cerda, Rodrigo. 2016. "Remuneraciones del sector público: ¿Mayores que en el sector privado?" Estudios Públicos 142: 8-35.

Gallegos, Sebastián, Alejandra Mizala \& Pilar Romaguera. 2011. "Public-private wage gap in Latin America (1992-2007): A matching approach". Labour Economics 18 (S1): 115-131.

Garloff, Alfred \& Anja Kuckulenz. 2006. "Training, mobility, and wages: Specific versus general human capital”. Journal of Economics and Statistics 226: 5581.

Jann Ben. 2008. "A Stata implementation of the Oaxaca-Blinder decomposition". ETH Zurich Sociology Working Paper 5.

Koenker, Roger \& Vasco D’Orey. 1987. “Computing regression quantiles”. Journal of the Royal Statistical Society 36 (3): 383-393. 
Melly, Blaise. 2005. "Public-private sector wage differentials in Germany: Evidence from quantile regression". Empirical Economics 30 (2): 505-520.

Mueller, Richard. 1998. "Public-private sector wage differentials in Canada: Evidence from quantile regressions". Economic Letters 60: 229-235.

Navarro, Lucas \& Javiera Selman. 2014. "Brechas salariales entre el sector público y privado en Chile: Evidencia a partir de datos longitudinales". Revista CEPAL 112: 94-110.

Oaxaca, Ronald. 1973. "Male-female wage differentials between the public and private sectors". International Economic Review 14: 693-709.

Robinson, Chris \& Nigel Tomes. 1984. "Union wage differentials in the public and private sectors: A simultaneous equations specification". Journal of Labor Economics 2 (1) 106-127.

\section{ANEXO}

En esta sección presentamos las tablas de resultados para las estimaciones que utilizan los datos de la encuesta Casen. Si bien se incorpora un cuadro sobre datos de la ESI, el resto de esas estimaciones se pueden solicitar a los autores.

Figura A1. PREMIO DEL SECTOR PÚBLICO EN SALARIO POR HORA (CASEN)

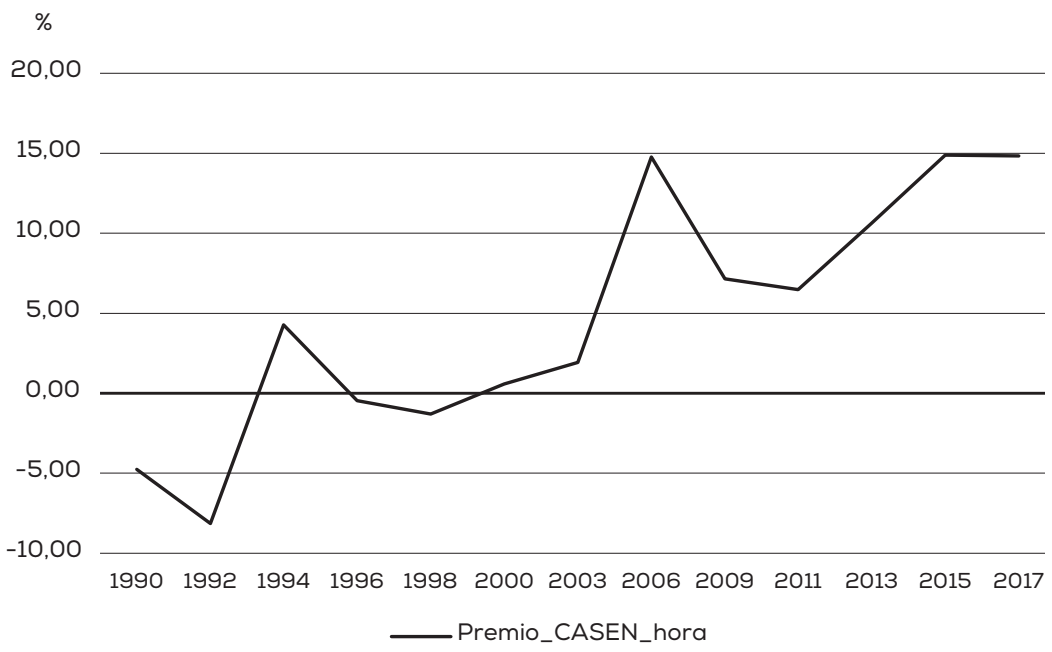


Cuadro A1. PREMIO DEL SECTOR PÚBLICO E INTERVALOS DE CONFIANZA AL 95 POR CIENTO (CASEN)

\begin{tabular}{lrcc}
\hline & & \multicolumn{2}{c}{ Intervalos de confianza } \\
\hline Año & Premio & Inferior & Superior \\
\hline 1990 & 1.51 & -4.58 & 7.61 \\
1992 & -6.05 & -10.93 & -1.16 \\
1994 & 4.01 & -1.49 & 9.52 \\
1996 & 2.27 & -3.88 & 8.42 \\
1998 & 1.57 & -3.41 & 6.55 \\
2000 & 3.97 & -0.29 & 8.22 \\
2003 & 7.27 & 3.86 & 10.67 \\
2006 & 7.57 & 3.32 & 11.48 \\
2009 & 12.87 & 8.80 & 16.94 \\
2011 & 12.15 & 7.41 & 16.90 \\
2013 & 17.61 & 11.41 & 23.82 \\
2015 & 19.52 & 16.80 & 22.24 \\
2017 & 20.04 & 17.29 & 22.80 \\
\hline
\end{tabular}

Cuadro A2. PREMIO DEL SECTOR PÚBLICO E INTERVALOS DE CONFIANZA AL 95 POR CIENTO (ESI)

\begin{tabular}{lccc}
\hline & \multicolumn{2}{c}{ Intervalos de confianza } \\
\hline Año & Premio & Inferior & Superior \\
\hline 2010 & 20.54 & 16.22 & 24.87 \\
2011 & 19.33 & 15.60 & 23.07 \\
2012 & 23.56 & 19.76 & 27.36 \\
2013 & 24.23 & 20.35 & 28.11 \\
2014 & 18.71 & 15.19 & 22.23 \\
2015 & 21.20 & 17.72 & 24.67 \\
2016 & 18.55 & 14.97 & 22.13 \\
2017 & 22.71 & 19.55 & 25.88 \\
\hline
\end{tabular}


|

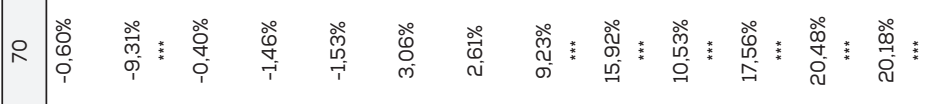

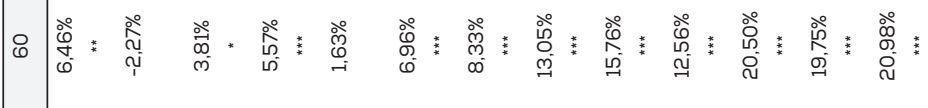

ம

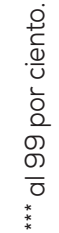

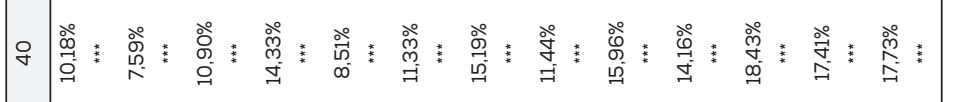

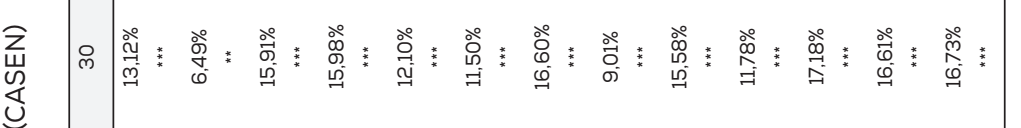

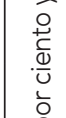

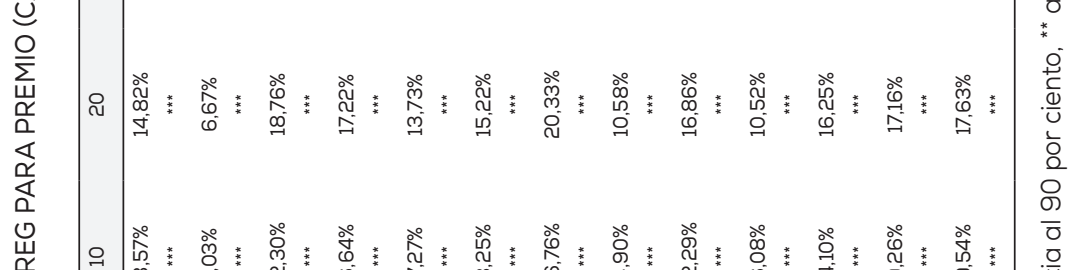

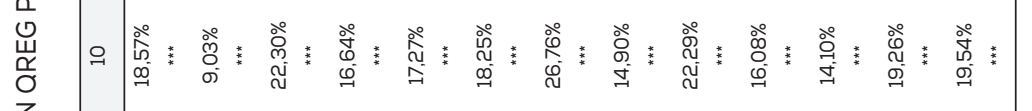

它

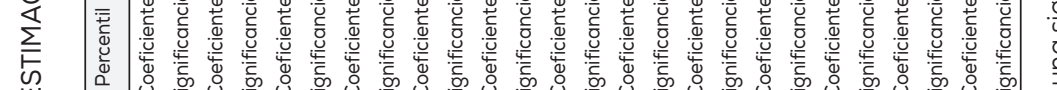

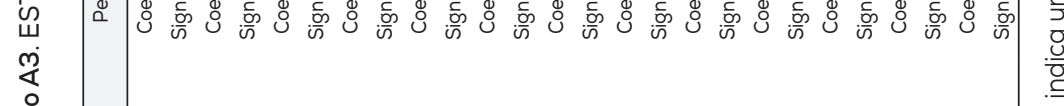

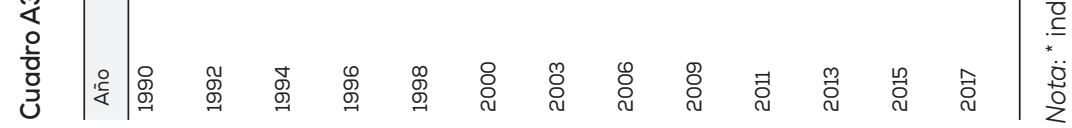


Cuadro A4. ESTIMACIÓN DESCOMPOSICIÓN DE BLINDER-OAXACA (CASEN)

\begin{tabular}{|c|c|c|c|c|}
\hline Año & Variable & Diferencia & Explicado & No explicado \\
\hline \multirow[t]{3}{*}{1990} & Coeficiente & 0,304 & 0,288 & 0,015 \\
\hline & Relevancia & & $94,92 \%$ & $5,08 \%$ \\
\hline & Significancia & $* * *$ & $* * *$ & \\
\hline \multirow[t]{3}{*}{1992} & Coeficiente & 0,229 & 0,288 & 0,059 \\
\hline & Relevancia & & $125,90 \%$ & $25,90 \%$ \\
\hline & Significancia & $* * *$ & $* * *$ & $* \star *$ \\
\hline \multirow[t]{3}{*}{1994} & Coeficiente & 0,409 & 0,368 & 0,041 \\
\hline & Relevancia & & $90,00 \%$ & $10,00 \%$ \\
\hline & Significancia & $* * *$ & $* * *$ & * \\
\hline \multirow[t]{3}{*}{1996} & Coeficiente & 0,298 & 0,275 & 0,023 \\
\hline & Relevancia & & $92,20 \%$ & $7,80 \%$ \\
\hline & Significancia & $* * *$ & $* * *$ & \\
\hline \multirow[t]{3}{*}{1998} & Coeficiente & 0,316 & 0,300 & 0,016 \\
\hline & Relevancia & & $94,97 \%$ & $5,03 \%$ \\
\hline & Significancia & $* * *$ & $* * *$ & \\
\hline \multirow[t]{3}{*}{2000} & Coeficiente & 0,237 & 0,197 & 0,040 \\
\hline & Relevancia & & $82,95 \%$ & $17,05 \%$ \\
\hline & Significancia & $* * *$ & $* * *$ & ** \\
\hline \multirow[t]{3}{*}{2003} & Coeficiente & 0,396 & 0,323 & 0,073 \\
\hline & Relevancia & & $81,67 \%$ & $18,33 \%$ \\
\hline & Significancia & $* * *$ & $* * *$ & $* * *$ \\
\hline \multirow[t]{3}{*}{2006} & Coeficiente & 0,314 & 0,241 & 0,074 \\
\hline & Relevancia & & $76,60 \%$ & $23,40 \%$ \\
\hline & Significancia & $* * *$ & $* * *$ & $* * *$ \\
\hline \multirow[t]{3}{*}{2009} & Coeficiente & 0,365 & 0,235 & 0,129 \\
\hline & Relevancia & & $64,57 \%$ & $35,43 \%$ \\
\hline & Significancia & $* \star *$ & $* * *$ & $* * *$ \\
\hline \multirow[t]{3}{*}{2011} & Coeficiente & 0,350 & 0,228 & 0,122 \\
\hline & Relevancia & & $65,13 \%$ & $34,87 \%$ \\
\hline & Significancia & $* * *$ & $* * *$ & $* \star *$ \\
\hline \multirow[t]{3}{*}{2013} & Coeficiente & 0,412 & 0,231 & 0,181 \\
\hline & Relevancia & & $56,09 \%$ & $43,91 \%$ \\
\hline & Significancia & $* * *$ & $* * *$ & $* \star *$ \\
\hline \multirow[t]{3}{*}{2015} & Coeficiente & 0,408 & 0,212 & 0,196 \\
\hline & Relevancia & & $51,96 \%$ & $48,04 \%$ \\
\hline & Significancia & $* * *$ & $* * *$ & $* \star *$ \\
\hline \multirow[t]{3}{*}{2017} & Coeficiente & 0,418 & 0,217 & 0,201 \\
\hline & Relevancia & & $51,99 \%$ & $48,01 \%$ \\
\hline & Significancia & $* * *$ & $* * *$ & $* \star *$ \\
\hline
\end{tabular}

Nota: * indica una significancia al 90 por ciento, ${ }^{* \star}$ al 95 por ciento $y$ *** al 99 por ciento. 